Bundesgesundheitsbl 2013 · 56:901-902 DOI 10.1007/s00103-013-1765-7

Online publiziert: 22. Juni 2013

๑) Springer-Verlag Berlin Heidelberg 2013

\section{J. Leidel ${ }^{1} \cdot 0$. Wichmann ${ }^{2}$}

${ }^{1}$ Vorsitzender der Ständigen Impfkommission, Geschäftsstelle der STIKO, Robert Koch-Institut, Berlin

${ }^{2}$ Leiter des Fachgebiets Impfprävention, Robert Koch-Institut, Berlin

\title{
Die Ständige Impfkommission und ihr neues methodisches Vorgehen
}

Die nach $\$ 20$ Abs. 2 Infektionsschutzgesetz (IfSG) beim Robert Koch-Institut eingerichtete Ständige Impfkommission (STIKO) hat den Auftrag, Empfehlungen zur Durchführung von Schutzimpfungen und zur Durchführung anderer Maßnahmen der spezifischen Prophylaxe übertragbarer Krankheiten beim Menschen in Deutschland zu geben. Sie wurde 1972 gegründet und besteht aktuell aus 17 ehrenamtlichen, persönlich berufenen Mitgliedern. Die Geschäftsstelle der STIKO ist am Robert Koch-Institut angesiedelt. Die STIKO-Empfehlungen dienen den obersten Landesgesundheitsbehörden als Grundlage für deren „öffentliche Empfehlungen“ für Schutzimpfungen gemäß $\$ 20$ Abs. 3 IfSG und unterstützen den öffentlichen Gesundheitsdienst und die Ärzteschaft bei der Durchführung von Schutzimpfungen. Die Empfehlungen sind ferner Grundlage für die Schutzimpfungsrichtlinie des Gemeinsamen Bundesausschusses.

Die STIKO gibt ihre Empfehlungen nach dem Stand der Wissenschaft und ist entsprechend ihrer Geschäftsordnung gehalten, ihr methodisches Vorgehen bei den erforderlichen Prüfungen und Bewertungen der verfügbaren Evidenz nach dem Stand der Wissenschaft festzulegen und zu veröffentlichen. Vor diesem Hintergrund hat die STIKO im November 2011 nach einem langjährigen, umfangreichen Diskussions- und Erarbeitungsprozess eine neue Methodik für die systematische Entwicklung von Impfempfehlungen verabschiedet, die als Standardvorgehensweise (Standard Opera- ting Procedure, SOP) auf ihren Internetseiten veröffentlicht ist (www.stiko.de).

Die STIKO führt bei der Erarbeitung von Impfempfehlungen in erster Linie eine Risiko-Nutzen-Bewertung sowohl auf individueller als auch auf Bevölkerungsebene durch. Als Leitlinie für die Entwicklung einer Impfempfehlung hat die STIKO einen Fragenkatalog erarbeitet, nach dem die notwendigen Daten für die Entscheidung über eine Impfempfehlung zusammengestellt werden. Dabei werden Daten zum Erreger und Krankheitsbild, zur Krankheitslast in Deutschland, zum Impfziel und zur Impfstrategie, zu Impfstoffwirksamkeit und -effektivität sowie zur erwarteten Akzeptanz der Impfung in der Bevölkerung berücksichtigt. Zusätzlich werden die möglichen Effekte der Impfung auf Bevölkerungsebene (z. B. Herdenschutz oder das Risiko für eine mögliche Verschiebung der Krankheitslast in andere Altersgruppen) berücksichtigt.

Bei der Bewertung von Impfstoffen und der Erarbeitung von Impfempfehlungen führt die STIKO systematische Übersichtsarbeiten (sog. Reviews) nach den Prinzipien der evidenzbasierten Medizin durch und bedient sich für die Erstellung ihrer Empfehlungen des Ansatzes der „Grading of Recommendations Assessment, Development and Evaluation" (GRADE) Working Group [1]. GRADE wird bereits international von diversen Kommissionen und Institutionen für entsprechende Fragestellungen benutzt, wie z. B. der US-amerikanischen Impfkommission ACIP oder der 
Impfkommission der Weltgesundheitsorganisation SAGE.

Mit finanzieller Unterstützung des Bundesministeriums für Gesundheit organisierte das Robert Koch-Institut 2010 und 2011 zwei internationale Workshops $\mathrm{zu}$ „Methoden für die Entwicklung von evidenzbasierten Impfempfehlungen“, an denen jeweils mehr als 40 Expertinnen und Experten aus dem In- und Ausland teilnahmen [2]. Unter anderem stellten Mitglieder von Impfkommissionen oder ihrer wissenschaftlichen Sekretariate Rahmenwerke bzw. Methoden vor, die bei der Entwicklung von Impfempfehlungen in den USA, Kanada, Holland, Finnland oder bei der Weltgesundheitsorganisation zur Anwendung kommen. Es bestand Einigkeit unter den Workshop-Teilnehmern, dass systematische und transparente Vorgehensweisen bei der Entwicklung von Impfempfehlungen notwendig sind, um ihre Qualität und öffentliche Akzeptanz zu optimieren. Dabei wurde auch erkannt, dass bei den systematischen Ansätzen der verschiedenen Impfkommissionen ähnliche Kriterien zur Anwendung kommen, was eine Nutzung von Synergien ermöglicht. So könnten z. B. systematische Übersichtsarbeiten zur Impfstoff-Effektivität und -Sicherheit oder mathematische Modelle zur Abschätzung von Impfeffekten gemeinsam erstellt oder ausgetauscht werden, um Doppelungen von zeitintensiven Tätigkeiten zu vermeiden. Das ist jedoch nur möglich, wenn aufgrund einer standardisierten Vorgehensweise und mit größtmöglicher Transparenz die Qualität dieser Arbeiten überprüft und die Einschätzung der Evidenzqualität nachvollzogen werden kann. GRADE wurde als die geeignete Methodik identifiziert, die dies ermöglicht.

Die neue Standardvorgehensweise der STIKO kam nun erstmals bei der Erarbeitung einer Empfehlung zur RotavirusImpfung zur Anwendung. Die verfügbare Evidenz, die den Entscheidungsprozess der STIKO unterstützte, ist in Form eines Hintergrundpapiers in dieser Ausgabe des Bundesgesundheitsblatts zusammengefasst. Dazu wurden mehrere systematische Reviews durchgeführt und die Qualität der Evidenz zur Wirksamkeit und Sicherheit der Impfstoffe mittels GRADE bewertet. Der Text wurde in englischer Sprache verfasst, um einen Austausch mit anderen nationalen oder internationalen Impfkommissionen zu unterstützen und eine kritische Begutachtung auch durch internationale Experten zuzulassen. Eine ausführliche Begründung der STIKOEmpfehlung in deutscher Sprache wird in gewohnter Weise im Epidemiologischen Bulletin des Robert Koch-Instituts erscheinen (Ausgabe 35/2013).

Die Autoren dieses Editorials möchten sich an dieser Stelle bei allen bedanken, die die Erarbeitung der neuen Standardvorgehensweise unterstützt haben, insbesondere dem Bundesministerium für Gesundheit, dem Gemeinsamen Bundesausschuss (insbesondere Herrn PD Dr. Perleth) sowie dem deutschen Cochrane Zentrum in Freiburg (insbesondere Herrn Prof. Dr. Antes und Herrn Dr. Meerpohl). Mit der neuen Standardvorgehensweise hat die STIKO den Entwicklungen in der evidenzbasierten Medizin Rechnung getragen und eine Methodik für die systematische Entwicklung von Impfempfehlungen nach dem aktuellen Stand der Wissenschaft etabliert. Die Autoren sind überzeugt davon, dass deren Anwendung sowohl die Qualität der Empfehlungen und die Transparenz der Entscheidungsprozesse steigern als auch einen Austausch mit nationalen und internationalen Partnern erleichtern wird.

Ihre

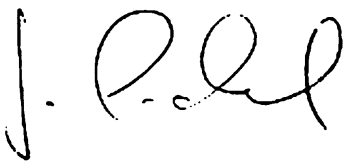

Jan Leidel

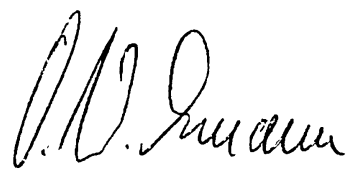

Ole Wichmann

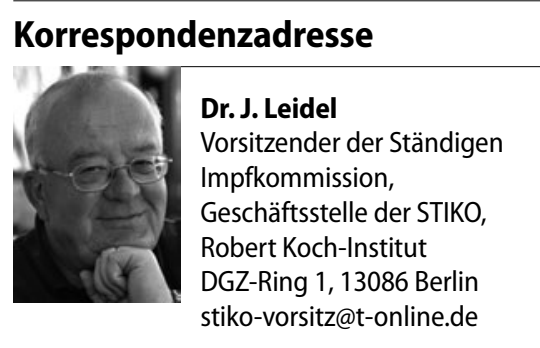

PD Dr. O. Wichmann
Leiter des Fachgebiets
Impfprävention,
Robert Koch-Institut
DGZ-Ring 1, 13086 Berlin
WichmannO@rki.de

\section{Literatur}

1. Guyatt $G H, O x m a n A D$, Vist GE, Kunz R, Falck-Ytter Y, Alonso-Coello P et al (2008) GRADE: an emerging consensus on rating quality of evidence and strength of recommendations. BMJ 336:924-926

2. Matysiak-Klose D, Ahmed F, Duclos P, Falck-Ytter Y, Forland F, Houweling $\mathrm{H}$ et al (2012) Report on the 1 st international workshop on procedures for the development of evidence-based vaccination recommendations, Berlin, Germany, 22-23 November 2010. Vaccine 30:2399-2404 